\title{
Immuno-Localization of CD44 and Osteopontin in Developing Human Kidney
}

\author{
GIOVANNA M. CRISI, SHARON A. MARCONI, GARY F. ROCKWELL, GREGORY L. BRADEN, AND THOMAS J. CAMPFIELD
}

Departments of Pathology [G.M.C., S.A.M.], Medicine [G.L.B.], and Pediatrics [G.F.R., T.J.C.], Baystate Medical Center, Springfield, MA 01199, Tufts University School of Medicine, Boston, MA 02111

\begin{abstract}
CD44 is observed in ureteric bud structures and is implicated in branching morphogenesis during early mouse renal development. Healthy adult kidney demonstrates minimal CD44, but CD44 is up-regulated in renal diseases. CD44 may mediate binding of calcium oxalate crystals to tubular epithelia via the ligands osteopontin (OPN) and hyaluronan. Because $15 \%$ of premature infants develop nephrocalcinosis, developmental tubular CD44 expression might promote nephrocalcinosis. We studied CD44 and OPN immuno-localization in developing human kidney by immunohistochemical analysis. Human renal tissue between 18 and 40 wk of gestation showed CD44 immuno-localization in ureteric buds, with staining decreasing with increasing gestational age; CD44 was rarely observed in developing renal tubules. OPN was diffusely observed in proximal tubules, rarely observed in distal tubules, ureteric buds or metanephric structures. These data support the role of CD44 in early human nephron formation and branching morphogenesis. Rare CD44 staining in developing tubular epithelium suggests no role for CD44 in promoting calcium oxalate adherence to tubular epithelia in premature infants. Immuno-localization of OPN in tubules supports its role in tubular differentiation, but OPN does not seem to be necessary during early nephron formation. (Pediatr Res 65: 79-84, 2009)
\end{abstract}

$\mathrm{P}$ remature birth imposes considerable stress on the kidney during a critical period of nephron formation and differentiation. Consequences of this stress include nephrocalcinosis, which has been reported to occur in approximately $15 \%$ of premature infants and may be associated with impaired renal function in later childhood (1-4). Decreased nephron mass in young adults who were born prematurely is associated with hypertension and impaired renal function (5). Studies of mammalian nephrogenesis have identified transcription factors, growth factors, and signaling proteins, which control the complex series of interactions resulting in kidney formation. Some factors that play a role in nephrogenesis can be reexpressed in adulthood during renal injury and may play a role in progressive renal damage (6). Although many of the basic mechanisms of nephrogenesis have been elucidated, there is very little information regarding immuno-localization of these factors in the developing human kidney.

CD44 is a cell surface glycoprotein receptor and adhesion molecule which is expressed in many human cell types and

Received February 20, 2008; accepted July 25, 2008.

Correspondence: Thomas J. Campfield, M.D., Newborn Medicine W2810, 759 Chestnut Street, Springfield, MA 01199; e-mail: thomas.campfield@bhs.org

Supported by BHS Incubator Grant 07-068, Baystate Medical Center.

Presented, in part, at the Annual meetings of the Pediatric Academic Societies and the American Society of Nephrology, 2007. has been implicated in a variety of physiologic and pathologic processes including lymphocyte homing, wound healing, cell migration, and tumor growth and metastasis (7-10). CD44 in normal mature human kidney is limited to endothelial cells, interstitial cells, and circulating leukocytes. However, upregulation of CD44 in tubular and glomerular epithelia because of renal injury has been observed in both human kidney disease and animal models of renal injury (11-16). CD44 is observed during renal embryogenesis at the ureteric bud, where reciprocal interaction of CD44 with hyaluronan (HA) plays a role in branching morphogenesis and early nephron differentiation (17-19). The CD44 ligand osteopontin (OPN) is an acidic glycoprotein, which has been characterized as a low-affinity, high-capacity calcium-binding protein (20). HA, also an important CD44 ligand, is a glycosaminoglycan abundant in renal medullary interstitium, is up-regulated in inflammatory renal disease and is a major crystal binding molecule at the surface of Madin-Darby canine kidney strain-1 cells $(21,22)$. Based on the affinity of these ligands for calcium, and the propensity for calcium oxalate to adhere to injured renal epithelia, a role for CD44 in promoting renal accumulation of calcium oxalate has been proposed. In this regard, Verhulst $e t$ al. (23) has suggested that preconditioning of renal epithelia by OPN and HA precedes the development of nephrocalcinosis in premature infants. However, no data are available regarding immuno-localization of CD44 in developing tubular epithelia.

This study was designed to examine the immunolocalization of CD44 and OPN in the developing human kidney between 18 and $40 \mathrm{wk}$ of gestation.

\section{METHODS}

This study was based on archival autopsy material and approved by the Institutional Review Board of Baystate Medical Center. Informed written consent was obtained at the time of autopsy with explicit understanding that tissue might be used for research without further need for consent. All cases were de-identified in compliance with HIPAA. The renal tissue studied was obtained after death because of intrauterine spontaneous fetal demise, or from infants who died because of the complications of prematurity. Fetal tissue was not studied in cases of elective termination of pregnancy.

A search of the pathology files from 1992 to 2006 was performed for autopsies between 18 and $40 \mathrm{wk}$ of intrauterine development. Hematoxylin and eosin stained kidney tissue sections were reviewed to evaluate for adequacy of tissue preservation based on the criteria of Genest (24) and for

Abbreviations: EMA, epithelial membrane antigen; HA, hyaluronan; OPN, osteopontin 
presence of an intact renal capsule. Exclusion criteria included genetic abnormalities (cytogenetic testing or morphology), and inadequate preservation of renal histomorphology. Control tissue included normal adult renal tissue obtained from neoplastic nephrectomy specimens $(n=4)$, and a failed renal allograft with acute rejection.

Immunohistochemistry and morphology. Immunohistochemical staining was performed on a DakoCytomation AutoStainer using the Signet Acuity Polymer Detection System HRP/DAB protocol in accordance with Signet Acuity data sheet (Dako, Carpenteria, CA). The primary antibodies used were: mouse anti-human MAb to PAN-CD44 (SFF-2, 1:10,000; Chemicon International, Temecula, CA); rabbit anti-human polyclonal antibody to OPN (aa75-90; 1:1500 dilutions; Chemicon International); mouse anti-human MAb to epithelial membrane antigen (anti-EMA) (E29, 1:3200; Dako). Tissue sections were cut at $4 \mu \mathrm{m}$ thickness, deparaffinized in xylene, cleared and rehydrated in graded ethanols as per standard procedures. Antigen retrieval was performed in a microwave using a $1 \times$ working concentration of Biogenex Concentrated $(10 \times)$ Antigen Retrieval Citra Solution Plus heated to and maintained at $98^{\circ} \mathrm{C}$ for $10 \mathrm{~min}$. Slides were incubated with the primary antibody for $30 \mathrm{~min}$, rinsed, and labeled polymer was applied for $25 \mathrm{~min}$. Antigen-antibody reaction was visualized using diaminobenzidine chromogen (DAB) applied for $7 \mathrm{~min}$, after which sections were counterstained in hematoxylin for $5 \mathrm{~min}$ and dehydrated in ethanol and in xylene before manual cover slipping. Positive and negative controls, omitting the primary antibody, were run in parallel.

In the subcapsular nephrogenic zone, CD44 and OPN immuno-localization were evaluated in areas beneath an intact capsule. For each renal tissue specimen, the percentage of CD44 and OPN positive vesicular bodies, C-shaped bodies, S-shaped bodies, and ureteric buds were calculated after the total number of these nephron structures was counted. The change in the percentage of positive structures as a function of gestational age (GA) was assessed using correlation, significance set at $p<0.05$.

In the cortical regions of the kidney, tubules were classified as proximal or distal based on morphology. Proximal tubules were identified by cuboidal to low columnar cells with abundant eosinophilic granular cytoplasm, centrally located nuclei, and an apical brush border. Distal tubules were identified by cuboidal and pale cells with scant cytoplasm, apically located nuclei and absent brush border. We assessed the reliability of these morphologic characteristics in developing kidneys by immunohistochemical EMA stain, which is specific for distal renal tubules (25). In the medulla, loop of Henle tubules were identified by small diameter and a low cuboidal or thin epithelial lining and absence of luminal red blood cells. Collecting ducts were identified by larger diameter and a cuboidal to columnar epithelial lining with well-defined cell borders. Proximal tubules, distal tubules, Henle's loop tubules, and collecting ducts were evaluated for CD44 and OPN immuno-localization using a semi-quantitative approach. To avoid bias, we examined 10 high power fields of representative regions of the entire renal tissue section. In each of these high power fields we counted proximal tubules, distal tubules, Henle's loop tubules, and collecting ducts, and identified these structures as positive or negative for CD44 and OPN. Having determined the percentage of CD44 and OPN positive structures in these 10 high power fields, each renal tissue section was classified using a four-tiered scoring system as follows: none: no tubules or ducts positive for CD44 or OPN; $1+:<10 \%$ of tubules or ducts positive; $2+$ : between $10 \%$ and $50 \%$ of tubules or ducts positive; or $3+:>50 \%$ of tubules or ducts positive.

\section{RESULTS}

We identified 43 cases between 18 and 40 wk GA for which tissue blocks were available and meeting the selection criteria. The cases were distributed as follows: 39 cases between 18 and $34 \mathrm{wk}$ GA with presence of nephrogenic zone, and four cases between 35 and 40 wk GA with absence of nephrogenic zone. The sex distribution was 13 female, 30 male. The cause of death in 38 cases was intrauterine fetal demise, whereas in five cases there was extra-uterine survival for up to $8 \mathrm{~d}$.

Differentiation of nephrogenic and tubular structures in the developing kidney. Figure $1 A$ demonstrates the histomorphology of the nephrogenic zone at $20 \mathrm{wk}$ of development with recognizable Wolffian duct and metanephric structures. Tubules that stained positive for EMA had morphologic characteristics of distal tubules, whereas EMA negative tubules had morphologic characteristics of proximal tubules (Fig. $2 A-C)$. Therefore, in sections stained for CD44 and OPN, classification of tubules as proximal or distal was based on morphologic criteria.

CD44 immuno-localization in developing human kidney. CD44 staining was not seen in normal adult renal tissue, whereas there was diffuse and strong CD44 staining of tubular epithelial cells in the rejected renal allograft (not shown). In the nephrogenic zone, immuno-localization of CD44 was seen in Wolffian duct-derived ureteric buds in all 39 tissue specimens between 18 and 34 wk GA (Fig. $1 B-E$ ), whereas metanephric structures (vesicular bodies, C-shaped bodies, Sshaped bodies) were negative for CD44. The percentage of CD44 positive ureteric buds among all cases ranged from 5 to $94 \%$ and decreased with increasing GA ( $p=0.02)$ (Fig. 3). Immuno-localization of CD44 in the medulla was minimal throughout development. CD44 immuno-localization is summarized in Table 1.

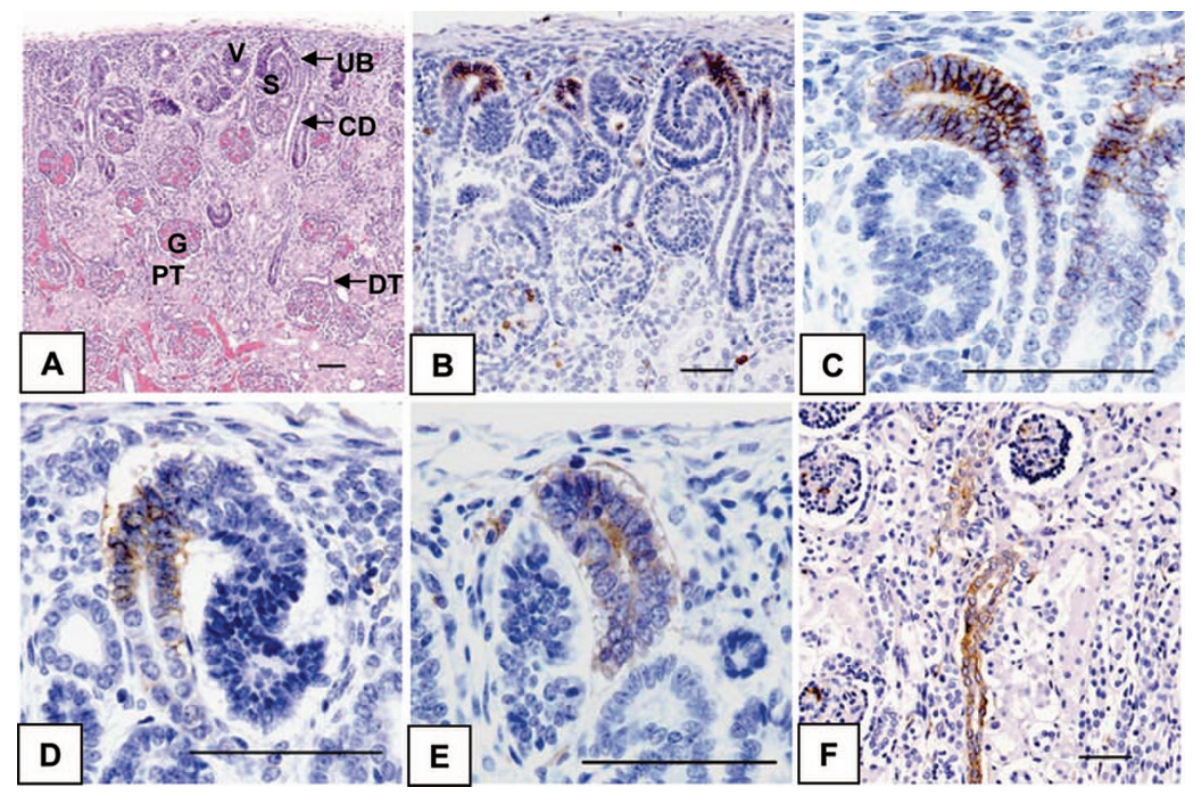

Figure 1. CD44 immuno-localization in developing human kidney. (A) Hematoxylin and eosin stained 20 wk gestational age (GA) kidney, showing different stages of renal development in the subcapsular nephrogenic zone. $(B-E)$ Immunohistochemical stain showing CD44 in a membranous and cytoplasmic pattern in ureteric bud (UB) structures in $20 \mathrm{wk}$ GA $(B-C), 26 \mathrm{wk}$ GA $(D)$ and 30 wk GA $(E)$, decreasing in intensity with advancing age. Metanephric structures including blastema, vesicle (V) and S-shaped structure (S), proximal tubule (PT), distal tubule (DT), and glomeruli (G) do not demonstrate CD44. Scattered CD44 positive endothelial cells and mononuclear cells are seen in $B$. $(F)$ CD44 staining is further reduced at 34 wk GA. CD, collecting duct. Scale bars $=50 \mu \mathrm{m}$. 


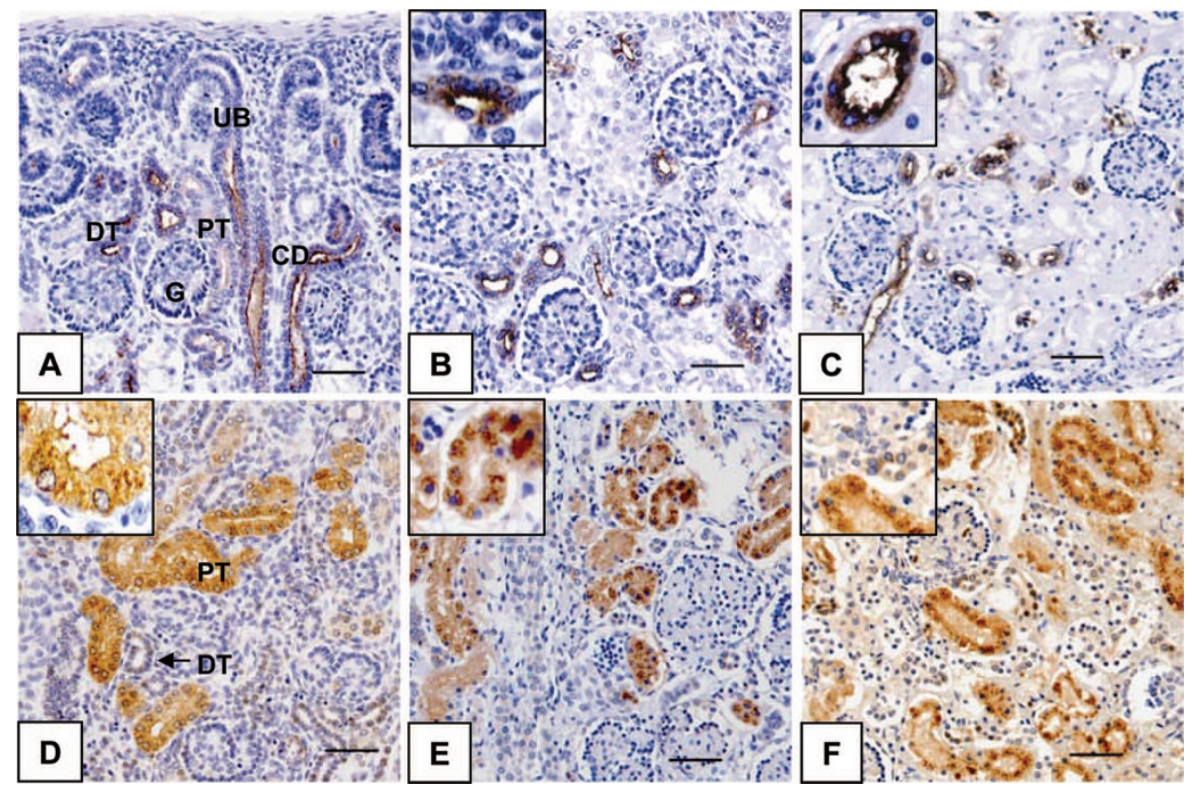

Figure 2. EMA and OPN immuno-localization in developing human kidney. (A) Immunohistochemical EMA apical and membranous staining of distal tubules (DT) and collecting ducts (CD) in 20-wk GA fetal kidney. (B) Higher power of A showing EMA positive distal tubule, some adjacent to glomerular hilum (insert). Proximal tubules (PT) and glomeruli (G) are EMA negative. (C) EMA staining in DT in $40 \mathrm{wk}$ GA, some adjacent to glomerular hilum (insert). $(D-F)$ OPN cytoplasmic and perinuclear staining (inserts) in proximal tubules in $20 \mathrm{wk} \mathrm{GA}(D), 30 \mathrm{wk} \mathrm{GA}(E)$, and 40 wk GA $(F)$. Insert in $F$ shows distal tubule at glomerular hilum with weak OPN cytoplasmic stain compared with PT (lower half). Scale bars = $50 \mu \mathrm{m}$.

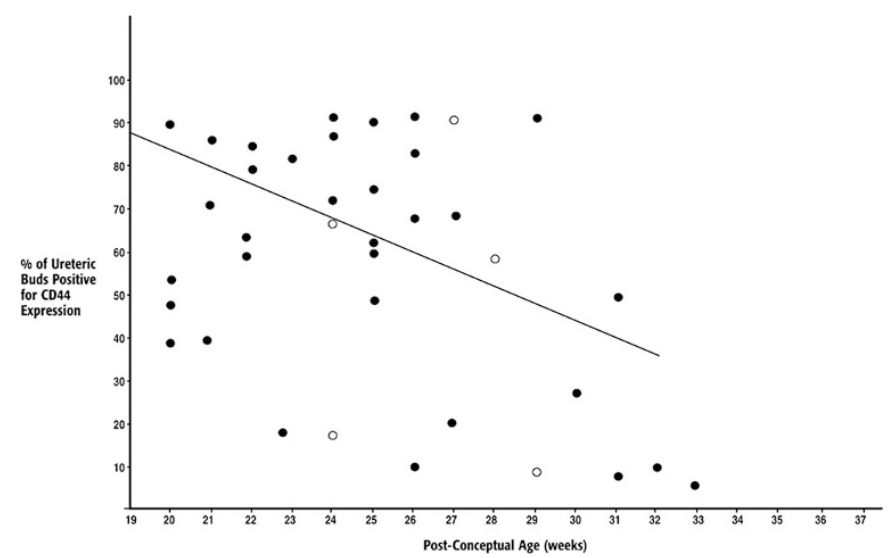

Figure 3. Correlation of ureteric bud CD44 staining with postconceptual age. $\circ$, infants who lived for up to $8 \mathrm{~d}$. $\bullet$, intrauterine demise. The percentage of ureteric buds staining positive for CD44 significantly decreased with increasing postconceptual age. $p<0.05$.

OPN immuno-localization in developing human kidney. All adult controls demonstrated OPN staining in $3+$ distal tubules in an apical plasma membrane pattern, and OPN staining of $2+$ proximal tubules in a perinuclear granular pattern (not shown). In the nephrogenic zone, there was no OPN staining in vesicular bodies, C-shaped bodies or Sshaped bodies at any GA. Ureteric bud immuno-localization of OPN was $1+$ in 12/43 (28\%) tissue specimens in a membranous pattern.

Between 18 and 40 wk of development, all tissue specimens were positive for OPN staining in proximal tubules. In 35/43 $(81 \%)$ of tissue specimens, there was perinuclear OPN staining in $3+$ proximal tubules (Fig. $2 D-F$ ), whereas $8 / 43(19 \%)$ of specimens had $1+$ or $2+$ OPN staining. In contrast, 14/43 specimens demonstrated no OPN staining of distal tubules. Remaining cases showed $1+$ and $2+$ distal tubules with apical membrane OPN staining in 11/43 and 10/43 tissue specimens, respectively. There was OPN staining in $3+$ distal tubules in only $8 / 43(19 \%)$ of tissue specimens. In the medulla, OPN staining was predominantly noted in loops of Henle. OPN staining patterns are summarized in Table 2.

We did not attempt to make statistical inferences about the effect of brief survival in the neonatal intensive care unit on the immuno-localization of theses factors, because of the small number of infants $(n=5)$ in this group.

\section{DISCUSSION}

We are the first to demonstrate the immuno-localization of CD44 in renal tissue from infants 18 to $40 \mathrm{wk} \mathrm{GA}$, representing the last periods of the metanephric phase of human kidney development (5). We show that CD44 immuno-localization is limited to Wolffian duct-derived ureteric buds in the nephrogenic zone until $32 \mathrm{wk} \mathrm{GA}$, after which ureteric buds become infrequent and disappear. CD44 was not observed in proximal tubules, rarely seen in distal tubules and never seen in early structures derived from metanephric blastema. These observations suggest that the role of CD44 in human renal development is limited to early mesenchymal-epithelial interaction, as has been reported in animal models (5,17-19). Our observation of CD44 immuno-localization in human fetal kidney ureteric bud suggests that the interaction of CD44 with HA or other potential ligands present in the surrounding mesenchyme may play a role in early human renal development. After 30 wk GA, CD44 staining was weak and noted in only a few ureteric buds, paralleling the exhaustion of metanephric mesenchyme and decrease in interstitial HA (17-19). These observations suggest that diminishing CD44 interactions with HA or other transcriptional target molecules such as Wnt in metanephric mesenchyme result in the termination of nephrogenesis (26).

A critical role for the CD44 ligand HA in renal development has been demonstrated using isolated ureteric buds and metanephric kidney cultures. Concentrations of HA below $1.5 \%$ at the ureteric bud tip promote branching and differentiation, whereas higher molecular weight and concentration inhibit branching. By this mechanism, local HA molecular 
Table 1. CD44 staining in developing human kidney between 20 and 40 weeks postconceptual age

\begin{tabular}{|c|c|c|c|c|c|c|}
\hline & $\begin{array}{l}\text { Percentage of renal } \\
\text { structures positive } \\
\text { for CD44 }\end{array}$ & $\begin{array}{l}18-24 \mathrm{Wk} \\
(n=18)\end{array}$ & $\begin{array}{l}25-29 \mathrm{Wk} \\
(n=15)\end{array}$ & $\begin{array}{l}30-33 \mathrm{Wk} \\
(n=6)\end{array}$ & $\begin{array}{l}34-40 \mathrm{Wk} \\
(n=4)\end{array}$ & $\begin{array}{c}\text { Total } \\
(n=43)\end{array}$ \\
\hline $\begin{array}{l}\text { Vesicular bodies, } \mathrm{C} \text {-shaped } \\
\text { and S-shaped bodies }\end{array}$ & None & \multicolumn{4}{|c|}{ No CD44 staining of vesicular, C-shaped or S-shaped bodies } & \\
\hline \multirow[t]{4}{*}{ Ureteric buds } & None & 0 & 0 & 3 & 4 & 7 \\
\hline & $1+$ & 0 & 0 & 0 & 0 & 0 \\
\hline & $2+$ & 5 & 3 & 3 & 0 & 11 \\
\hline & $3+$ & 13 & 12 & 0 & 0 & 25 \\
\hline Proximal tubules & None & \multicolumn{4}{|c|}{ No CD44 staining of proximal tubules } & \\
\hline \multirow[t]{4}{*}{ Distal tubules } & None & 14 & 10 & 4 & 1 & 29 \\
\hline & $1+$ & 4 & 5 & 0 & 3 & 12 \\
\hline & $2+$ & 0 & 0 & 2 & 0 & 2 \\
\hline & $3+$ & 0 & 0 & 0 & 0 & 0 \\
\hline \multirow[t]{4}{*}{ Collecting ducts } & None & 15 & 10 & 5 & 2 & 32 \\
\hline & $1+$ & 3 & 5 & 1 & 2 & 11 \\
\hline & $2+$ & 0 & 0 & 0 & 0 & 0 \\
\hline & $3+$ & 0 & 0 & 0 & 0 & 0 \\
\hline \multirow[t]{4}{*}{ Loop of Henle } & None & 12 & 11 & 5 & 3 & 31 \\
\hline & $1+$ & 6 & 4 & 1 & 1 & 12 \\
\hline & $2+$ & 0 & 0 & 0 & 0 & 0 \\
\hline & $3+$ & 0 & 0 & 0 & 0 & 0 \\
\hline
\end{tabular}

Percent of renal structures positive for CD44 expressed as follows: none, no structures positive; $1+,<10 \%$ of structures positive; $2+, 10-50 \%$ of structures positive; $3+,>50 \%$ of structures positive.

Table 2. Osteopontin staining in developing human kidney between 20 and 40 weeks postconceptual age

\begin{tabular}{|c|c|c|c|c|c|c|}
\hline & $\begin{array}{l}\text { Percentage of renal } \\
\text { structures positive } \\
\text { for osteopontin }\end{array}$ & $\begin{array}{c}18-24 \mathrm{Wk} \\
(n=18)\end{array}$ & $\begin{array}{l}25-29 \mathrm{Wk} \\
(n=15)\end{array}$ & $\begin{array}{l}30-33 \mathrm{Wk} \\
(n=6)\end{array}$ & $\begin{array}{l}34-40 \mathrm{Wk} \\
\quad(n=4)\end{array}$ & $\begin{array}{c}\text { Total } \\
(n=43)\end{array}$ \\
\hline $\begin{array}{l}\text { Vesicular bodies, C-shaped } \\
\text { and S-shaped bodies }\end{array}$ & None & \multicolumn{5}{|c|}{$\begin{array}{l}\text { No osteopontin staining of vesicular, C-shaped or S-shaped bodies between } 20 \\
\text { and } 40 \mathrm{wk} \text { of development }\end{array}$} \\
\hline \multirow[t]{4}{*}{ Ureteric buds } & None & 14 & 11 & 2 & 4 & 31 \\
\hline & $1+$ & 4 & 4 & 4 & 0 & 12 \\
\hline & $2+$ & 0 & 0 & 0 & 0 & 0 \\
\hline & $3+$ & 0 & 0 & 0 & 0 & 0 \\
\hline \multirow[t]{4}{*}{ Proximal tubules } & None & 0 & 0 & 0 & 0 & 0 \\
\hline & $1+$ & 1 & 0 & 0 & 0 & 1 \\
\hline & $2+$ & 2 & 4 & 1 & 0 & 7 \\
\hline & $3+$ & 15 & 11 & 5 & 4 & 35 \\
\hline \multirow[t]{4}{*}{ Distal tubules } & None & 10 & 2 & 2 & 0 & 14 \\
\hline & $1+$ & 5 & 4 & 2 & 0 & 11 \\
\hline & $2+$ & 1 & 4 & 2 & 3 & 10 \\
\hline & $3+$ & 2 & 5 & 0 & 1 & 8 \\
\hline \multirow[t]{4}{*}{ Collecting ducts } & None & 10 & 12 & 6 & 4 & 32 \\
\hline & $1+$ & 4 & 2 & 0 & 0 & 6 \\
\hline & $2+$ & 4 & 1 & 0 & 0 & 5 \\
\hline & $3+$ & 0 & 0 & 0 & 0 & 0 \\
\hline \multirow[t]{4}{*}{ Loop of Henle } & None & 2 & 0 & 1 & 0 & 3 \\
\hline & $1+$ & 8 & 4 & 3 & 2 & 17 \\
\hline & $2+$ & 2 & 5 & 0 & 1 & 8 \\
\hline & $3+$ & 6 & 6 & 2 & 1 & 15 \\
\hline
\end{tabular}

Percent of renal structures positive for osteopontin expressed as follows: none, no structures positive; $1+,<10 \%$ of structures positive; $2+, 10-50 \%$ of structures positive; $3+,>50 \%$ of structures positive.

weight and concentration may promote ureteric bud branching early in development, and bring about the cessation of branching at the appropriate time. Local differences in HA molecular weight and concentration may be regulated via developmental and topographic expression of enzymes responsible for HA synthesis and degradation (27). These observations suggest a critical role for HA during early renal development. Because CD44 is the principle HA cell surface receptor, our observations of CD44 at the ureteric bud tip suggest a role for CD44 in human renal development. However, a developmental decrease in the number of CD44-positive ureteric buds does not necessarily lend substantial support to a critical role for CD44 in early epithelial-mesenchymal interaction. Indeed, mice targeted for deletion of the CD44 gene develop grossly normal kidneys $(28,29)$. The CD44 ligand HA is diffusely present in the developing human kidney. Verhulst et al. (23) have shown that HA is present in the interstitium of fetal kidneys throughout the period of nephrogenesis, decreasing along with inter- 
stitial volume as tubular growth occurs, and also observed HA at the luminal surface of developing tubules. Perhaps CD44-HA interaction regulates renal ureteric bud branching by promoting or inhibiting movement of other signaling molecules to the tips of the ureteric bud.

Although CD44 immuno-localization in developing tubules was rare, we observed diffuse OPN staining in developing tubules, especially proximal tubules. Animal studies have demonstrated the importance of OPN in tubule development. OPN is up-regulated in tubules of rat embryos with advancing development, and OPN inhibition by anti-OPN antibody disrupts tubule development (30-32). The ontogeny of OPN in human renal development has been reported in two studies. Hudkins et al. (33) examined fetuses between seven and 18 wk GA and observed that before $80 \mathrm{~d}$ of development OPN staining is weak and localized to the luminal surface of some tubules and some ureteric buds. After $80 \mathrm{~d}$ of development, increased OPN staining was noted throughout the tubular cytoplasm. Verhulst et al. (23) demonstrated OPN staining of cortical and medullary tubules in infants between 24 and 40 wk GA. These reports did not address differential staining of OPN in proximal and distal tubules. In cultures of adult human proximal and distal tubules, different staining patterns were noted by immunocytochemical and confocal microscopy (34). Perinuclear golgi apparatus OPN staining was noted in proximal tubules, whereas apical plasma membrane OPN staining was noted in distal tubules.

We were able to differentiate proximal and distal tubules as early as 18 wk GA and we observed most OPN staining in proximal rather than distal tubules during early human renal development. We observed OPN immuno-localization in EMA negative proximal tubules in a perinuclear granular pattern, whereas EMA positive distal tubules demonstrated apical plasma membrane staining. In mature normal kidney, OPN is present in distal tubules in an apical plasma membrane pattern suggesting export of OPN into the tubular lumen (35). In contrast, the normal adult kidney displays little or no proximal tubule staining of OPN. However, strong proximal tubule OPN staining in a cytoplasmic pattern is seen in diabetic kidney, acute allograft rejection and cyclosporine toxicity (36-40). These patterns of OPN staining suggest that OPN up-regulation in proximal tubules occurs in response to injury. In our studies, we noted OPN staining pattern in developing kidney similar to that seen in adult renal injury. It is tempting to speculate that proximal tubule OPN staining in injured adult kidney represents a re-appearance of the fetal pattern of OPN expression.

We have shown that premature infants have decreased urinary OPN concentration compared with adults (41). Our current study suggests minimal synthesis and cellular export of OPN into cortical distal tubular segments where there is a high concentration of stone-forming constituents. The lack of OPN staining at this distal nephron site may be in agreement with our data showing decreased urinary excretion of OPN in premature infants.

Damaged and regenerating tubular epithelial cells have a propensity to bind calcium oxalate crystals (42). Binding of CD44 to OPN or HA has been shown to promote calcium oxalate crystal adherence to Madin-Darby canine kidney cells (22). Asselman and coworkers have proposed a model of nephrocalcinosis in which CD44, up-regulated in injured epithelia, binds OPN or HA, which in turn bind calcium oxalate crystals in the tubular lumen $(43,44)$. Verhulst et al. (23) emphasized the importance of OPN in the pathogenesis of nephrocalcinosis in premature infants, pointing out that presence of OPN precedes the development of nephrocalcinosis in these infants. In our study, we noted only rare $(<10 \%$ of distal tubules) CD44 staining in $35 \%$ of tissue samples, whereas $65 \%$ of these tissue specimens demonstrated no tubular CD44 staining at all. Thus, our observations do not support a critical role for constitutive CD44 expression in the pathogenesis of nephrocalcinosis in premature infants. It is possible that premature infants who suffer repeated bouts of hypoxemia, hypotension, and infection have up-regulation of tubular CD44 expression. It is likely that the etiology of nephrocalcinosis in premature infants is multi-factorial, with developmental, pharmacologic, and nutritional factors contributing to calcium oxalate accumulation.

In summary, we demonstrate the immuno-localization of CD44 in developing kidney between 18 and 40 wk of human development. CD44 staining is seen in ureteric bud epithelium in the subcapsular nephrogenic zone, with diminishing staining as nephron formation progresses to completion. These observations lend support to the concept that CD44 plays a role in early epithelial-mesenchymal interactions. Lack of widespread CD44 immuno-localization in developing tubules does not support the concept that developmental CD44 expression plays a critical role in the pathogenesis of nephrocalcinosis in premature infants. Our data do not however address the possibility of renal CD44 up-regulation in sick premature infants. We demonstrate that distal tubules can be differentiated from other nephron segments by EMA immunoreactivity and Hematoxylin and eosin morphology. In premature infants, OPN immuno-localization mainly occurs in proximal tubules in a cytoplasmic and perinuclear granular pattern early in human development with less staining in distal tubules. This pattern of OPN immuno-localization may play a role in the development of nephrocalcinosis in premature infants.

Acknowledgment. We thank James D. Mueller, M.D. for constructive advice during the preparation of this manuscript.

\section{REFERENCES}

1. Hein G, Richter D, Manz F, Weitzel D, Kalhoff H 2004 Development of nephrocalcinosis in very low birth weight infants. Pediatr Nephrol 19:616-620

2. Campfield T, Braden G, Flynn-Valone P, Clark N 1994 Urinary oxalate excretion in premature infants: effect of human milk versus formula feeding. Pediatrics 94:674678

3. Kist-van Holthe JE, van Zwieten PH, Schell-Feith EA, Zonderland HM, Holscher HC, Wolterbeek R, Veen S, Frolich M, van der Heijden BJ 2007 Is nephrocalcinosis in preterm neonates harmful for long-term blood pressure and renal function? Pediatrics 119:468-475

4. Ezzedeen F, Adelman RD, Ahlfors CE 1988 Renal calcification in preterm infants: pathophysiology and long-term sequelae. J Pediatr 113:532-539

5. Clark AT, Bertram JF 1999 Molecular regulation of nephron endowment. Am J Physiol 276:F485-F497

6. Hershkovitz D, Burbea Z, Skorecki K, Brenner BM 2007 Fetal programming of adult kidney disease: cellular and molecular mechanisms. Clin J Am Soc Nephrol 2:334342 
7. Günthert U, Hofmann M, Rudy W, Reber S, Zoller M, Haussmann I, Matzku S, Wenzel A, Ponta H, Herrlich P 1991 A new variant of glycoprotein CD44 confers metastatic potential to rat carcinoma cells. Cell 65:13-24

8. Goodison S, Urquidi V, Tarin D 1999 CD44 cell adhesion molecules. Mol Pathol $52: 189-196$

9. Nagano O, Saya H 2004 Mechanism and biological significance of CD44 cleavage. Cancer Sci 95:930-935

10. Mackay CR, Terpe HJ, Stauder R, Marston WL, Stark H, Günthert U 1994 Expression and modulation of CD44 variant isoforms in humans. J Cell Biol 124:71-82

11. Wuthrich RP 1999 The proinflammatory role of hyaluronan-CD44 interactions in renal injury. Nephrol Dial Transplant 14:2554-2556

12. Lewington AJ, Padanilam BJ, Martin DR, Hammerman MR 2000 Expression of CD44 in kidney after ischemic injury in rats. Am J Physiol Regul Integr Comp Physiol 278:R247-R254

13. Benz PS, Fan X, Wüthrich RP 1996 Enhanced tubular epithelial CD44 expression in MRL-lpr lupus nephritis. Kidney Int 50:156-163

14. Sibalic V, Fan X, Loffing J, Wüthrich RP 1997 Upregulated renal tubular CD44, hyaluronan, and osteopontin in kdkd mice with interstitial nephritis. Nephrol Dial Transplant 12:1344-1353

15. Florquin S, Nunziata R, Claessen N, van den Berg FM, Pals ST, Weening JJ 2002 CD44 expression in IgA nephropathy. Am J Kidney Dis 39:407-414

16. Rouschop KM, Roelofs JJ, Sylva M, Rowshani AT, Ten Berge IJ, Weening JJ, Florquin S 2006 Renal expression of CD44 correlates with acute renal allograft rejection. Kidney Int 70:1127-1134

17. Pohl M, Sakurai H, Stuart RO, Nigam SK 2000 Role of hyaluronan and CD44 in in vitro branching morphogenesis of ureteric bud cells. Dev Biol 224:312-325

18. Pohl M, Stuart RO, Sakurai H, Nigam SK 2000 Branching morphogenesis during kidney development. Ann Rev Physiol 62:595-620

19. Piscione TD, Rosenblum ND 2002 The molecular control of renal branching morphogenesis: current knowledge and emerging insights. Differentiation 70:227246

20. Chen Y, Bal BS, Gorski JP 1992 Calcium and collagen binding properties of osteopontin, bone sialoprotein, and bone acidic glycoprotein-75 from bone. J Biol Chem 267:24871-24878

21. Aruffo A, Stamenkovic I, Melnick M, Underhill CB, Seed B 1990 CD44 is the principal cell surface receptor for hyaluronate. Cell 61:1303-1313

22. Verkoelen CF, van der Boom BG, Romijn JC 2000 Identification of hyaluronan as a crystal-binding molecule at the surface of migrating and proliferating MDCK cells Kidney Int 58:1045-1054

23. Verhulst A, Asselman M, de Naeyer B, Vervaet BA, Mengel M, Gwinner W, D'Haese PC, Verkoelen CF, de Broe ME 2005 Preconditioning of the distal tubular epithelium of the human kidney precedes nephrocalcinosis. Kidney Int 68:16431647

24. Genest DR, Williams MA, Greene MF 1992 Estimating the time of death in stillborn fetuses. I. Histologic evaluation of fetal organs; an autopsy study of 150 stillborns. Obstet Gynecol 80:575-584

25. Fleming S, Lindop GB, Gibson AA 1985 The distribution of epithelial membrane antigen in the kidney and its tumours. Histopathology 9:729-739

26. Merkel CE, Karner CM, Carroll TJ 2007 Molecular regulation of kidney development: is the answer blowing in the Wnt? Pediatr Nephrol 22:1825-1838

27. Rosines E, Schmidt HJ, Nigam SK 2007 The effect of hyaluronic acid size and concentration on branching morphogenesis and tubule differentiation in developing kidney culture systems: potential applications to engineering of renal tissues. Biomaterials 28:4806-4817
28. Rouschop KM, Sewnath ME, Claessen N, Roelofs JJ, Hoedemaeker I, van der Neut R, Aten J, Pals SJ, Weening JJ, Florquin S 2004 CD44 deficiency increases tubula damage but reduces renal fibrosis in obstructive nephropathy. J Am Soc Nephro 15:674-686

29. Herrera MB, Bussolati B, Bruno S, Morando L, Mauriello-Romanazzi G, Sanavio F, Stamenkovic I, Biancone L, Camussi G 2007 Exogenous mesenchymal stem cells localize to the kidney by means of CD44 following acute tubular injury. Kidney Int 72:430-441

30. Denda S, Reichardt LF, Müller U 1998 Identification of OPN as a novel ligand for the integrin alpha8 beta1 and potential roles for the integrin-ligand interaction in kidney morphogenesis. Mol Biol Cell 9:1425-1435

31. Nomura S, Wills AJ, Edwards DR, Heath JK, Hogan BL 1988 Developmenta expression of 2 ar (osteopontin) and SPARC (osteonectin) RNA as revealed by in situ hybridization. J Cell Biol 106:441-450

32. Rogers SA, Padanilam BJ, Hruska KA, Giachelli CM, Hammerman MR 1997 Metanephric osteopontin regulates nephrogenesis in vitro. Am J Physiol 272:F469F476

33. Hudkins KL, Gianchelli CM, Cui Y, Couser WG, Johnson RJ, Alpers CE 1999 Osteopontin expression in fetal and mature human kidney. J Am Soc Nephro 10:444-457

34. Verhulst A, Persy VP, van Rompay AR, Verstrepen WA, Helbert MF, de Broe ME 2002 Osteopontin synthesis and localization along the human nephron. J Am Soc Nephrol 13:1210-1218

35. Xie Y, Sakatsume M, Nishi S, Narita I, Arakawa M, Gejyo F 2001 Expression, roles, receptors, and regulation of osteopontin in the kidney. Kidney Int 60:1645-1657

36. Fischer JW, Tschöpe C, Reinecke A, Giachelli CM, Unger T 1998 Upregulation of osteopontin expression in renal cortex of streptozotocin-induced diabetic rats is mediated by bradykinin. Diabetes 47:1512-1518

37. Kelly DJ, Wilkinson-Berka JL, Ricardo SD, Cox AJ, Gilbert RE 2002 Progression of tubulointerstitial injury by osteopontin-induced macrophage recruitment in advanced diabetic nephropathy of transgenic (mRen-27) rats. Nephrol Dial Transplan 17:985-991

38. Yu XQ, Wu LL, Huang XR, Yang N, Gilbert RE, Cooper ME, Johnson RJ, Lai KN Lan HY 2000 Osteopontin expression in progressive renal injury in remnant kidney: role of angiotensin II. Kidney Int 58:1469-1480

39. Alchi B, Nishi S, Kondo D, Kaneko Y, Matsuki A, Imai N, Ueno M, Iguchi S, Sakatsume M, Narita I, Yamamoto T, Gejyo F 2005 Osteopontin expression in acute renal allograft rejection. Kidney Int 67:886-896

40. Hudkins KL, Le QC, Segerer S, Johnson RJ, Davis CL, Giachelli CM, Alpers CE 2001 Osteopontin expression in human cyclosporine toxicity. Kidney Int 60:635640

41. Rockwell GF, Morgan MJ, Braden G, Campfield TJ 2008 Preliminary observation of urinary calcium and osteopontin excretion in premature infants, term infants and adults. Neonatology 93:241-245

42. Verkoelen CF, van der Boom BG, Houtsmuller AB, Schröder FH, Romijn JC 1998 Increased calcium oxalate monohydrate crystal binding to injured renal tubular epithelial cells in culture. Am J Physiol 274:F958-F965

43. Asselman M, Verhulst A, de Broe ME, Verkoelen CF 2003 Calcium oxalate crystal adherence to hyaluron-, osteopontin-, and CD44-expressing injured/regenerating tubular epithelial cells in rat kidneys. J Am Soc Nephrol 14:3155-3166

44. Verhulst A, Asselman M, Persy VP, Schepers MS, Helbert MF, Verkoelen CF, de Broe ME 2003 Crystal retention capacity of cells in the human nephron: involvement of CD44 and its ligands hyaluronic acid and osteopontin in the transition of a crystal-binding into a nonadherent epithelium. J Am Soc Nephrol 14:107-115 